\title{
en
}

\section{Electromagnetic Wormholes and Virtual Magnetic Monopoles from Metamaterials}

\author{
Allan Greenleaf* \\ Department Mathematics University of Rochester, Rochester, New York 14627, USA \\ Yaroslav Kurylev \\ Department of Mathematical Sciences, University College London, Gower Str, London, WC1E 6BT, United Kingdom \\ Matti Lassas \\ Institute of Mathematics, Helsinki University of Technology, FIN-02015, Finland \\ Gunther Uhlmann \\ Department of Mathematics, University of Washington, Seattle, Washington 98195, USA
}

(Received 15 March 2007; published 29 October 2007)

\begin{abstract}
We describe new configurations of electromagnetic (EM) material parameters, the electric permittivity $\epsilon$ and magnetic permeability $\mu$, which allow one to construct devices that function as invisible tunnels. These allow EM wave propagation between the regions at the two ends of a tunnel, but the tunnels themselves and the regions they enclose are not detectable to lateral EM observations. Such devices act as wormholes with respect to Maxwell's equations and effectively change the topology of space vis-à-vis EM wave propagation. We suggest several applications, including devices behaving as virtual magnetic monopoles, invisible cables, and scopes for MRI-assisted surgery.
\end{abstract}

DOI: 10.1103/PhysRevLett.99.183901

PACS numbers: 42.70.Qs, 41.20.Jb, 42.79.Ry

Introduction. - New custom designed electromagnetic (EM) media, or metamaterials, have inspired plans to create cloaking, or invisibility, devices that would render objects located within invisible to observation by exterior measurements of EM waves [1-5]. Such a device is theoretically described by means of an "invisibility coating," consisting of a medium whose EM material parameters (the electric permittivity $\epsilon$ and magnetic permeability $\mu$ ) are designed to manipulate EM waves in a way that is not encountered in nature. Experimentally, cloaking has now been implemented with respect to microwaves in [6], with the invisibility coating consisting of metamaterials fabricated and assembled to approximate the desired ideal fields $\epsilon$ and $\mu$ at $8.5 \mathrm{GHz}$. Mathematically, these constructions have their origin in singular changes of coordinates; similar analysis in the context of electrostatics (or its mathematical equivalent) is already in [7-11]. Versions for elasticity are in $[12,13]$.

Potential applications of the cloaking constructions are limited by the lack of interaction between objects and EM waves in the cloaked region and those in the exterior region. It would be desirable to allow some limited interaction, with the nature and quantity controlled by design parameters. In this Letter, we show that more elaborate geometric ideas than those in cloaking enable the construction of devices, i.e., the specification of ideal $\epsilon$ and $\mu$, that function as EM wormholes, allowing the passage of waves between possibly distant regions while most of the region of propagation remains invisible. At a noncloaking frequency, the resulting construction appears (roughly) as a solid cylinder, but at cloaking frequencies, i.e., $k$ for which $\epsilon$ and $\mu$ are effective, the wormhole device has the effect of changing the topology of space. EM waves propagate as if $\mathbb{R}^{3}$ has a handlebody attached to it (see 2-dimensional schematic in Fig. 1). Any object inside the handlebody is only visible to waves which enter from either of the ends; conversely, EM waves propagating inside the wormhole, say from a source, can only leave through the ends. Thus, for example, a magnetic dipole situated near one end of the wormhole would appear to an external observer as a magnetic monopole. Already on the level of ray-tracing, the wormhole construction gives rise to interesting effects (Fig. 2). We conclude by describing other possible applications of wormhole devices. A detailed analysis of wormholes is given elsewhere [14].

Background.-Mathematically, most of the cloaking constructions mentioned above have their origins in a singular transformation of space in which an infinitesimally small hole has been stretched to a ball (the boundary of which is the cloaking surface). An object can then be

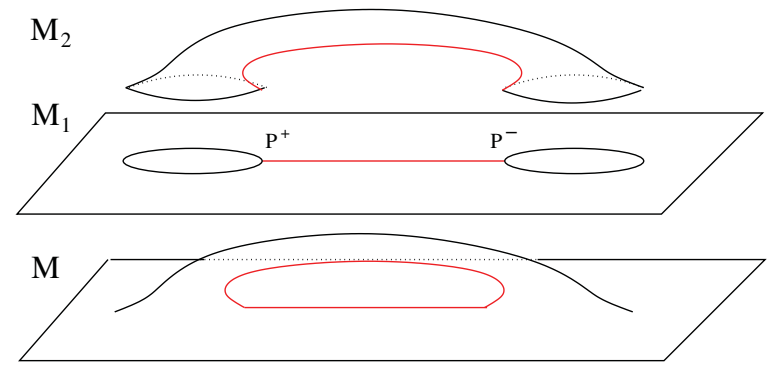

FIG. 1 (color). Schematic figure: a wormhole manifold is glued from two components, the "handle" and space with two holes. In the actual construction, components are 3-dimensional. Red curves on $M_{1}, M_{2}$, and $M$ are $\gamma_{1}, \gamma_{2}$, and $\gamma$, respectively. 

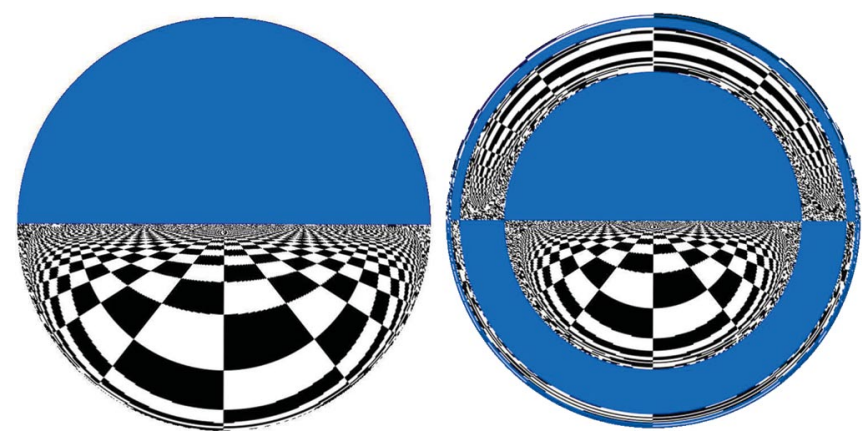

FIG. 2 (color). Ray-tracing simulations of views through the bores of two wormholes. The distant ends are above an infinite chess board under a blue sky. On left, $L \ll 1$; on right, $L \approx 1$. Note that the blue is used for clarity; the wormhole construction should be considered essentially monochromatic.

inserted inside the hole so created and made invisible to external observations. We call this process blowing up a point. The cloaking effect of such singular transformations was justified in $[1,2]$ both on the level of the chain rule on the exterior of the cloaked region, where the transformation is smooth, as well as on the level of ray-tracing in the exterior.

However, due to the singular nature of the resulting EM parameters, to fully justify this construction, one needs to study physically meaningful solutions of the resulting (degenerate) Maxwell's equations on all of space, including the cloaked region and particularly at the cloaking surface itself. These are waves that are both finite energy and distributional solutions, i.e., that satisfy Maxwell in an appropriate weak sense.

This was carried out in [5], and it was shown that the original cloaking constructions in dimension 3 are indeed valid; furthermore, EM active objects (those with nontrivial internal currents) may be cloaked as well, if the interior of the cloaking surface is appropriately lined. Although the analysis works at all frequencies $k$, the cloaking effect should be considered as essentially monochromatic, or at best narrow band, using current technology, since the metamaterials needed to physically implement these ideal constructions are subject to significant dispersion [2]. These same considerations hold for the wormhole constructions described here.

The wormhole manifold M. - First, we explain what we mean by a wormhole. The concept is familiar from cosmology [15], but here we use the term to denote a specification of $\varepsilon$ and $\mu$ giving rise to certain effects. We start by describing the abstraction of this process on the level of what we call the wormhole manifold and then explain how this can be effectively realized vis-à-vis EM wave propagation by giving EM parameter fields $\epsilon$ and $\mu$ in a region $N \subset \mathbb{R}^{3}$, the wormhole device. Together, these can be considered as "blowing up a curve," rather than the "blowing up a point" used in cloaking. Employing metamaterials, it should be possible to physically construct a device having EM parameters at least approximating the ideal wormhole and thereby experimentally produce the effects that we describe.

Let us start by making two holes in the Euclidian space $\mathbb{R}^{3}$, say by removing the open unit balls $B^{-}$and $B^{+}$, separated by some positive distance. Denote by $M_{1}$ the region so obtained, $M_{1}=\mathbb{R}^{3} \backslash\left(B^{-} \cup B^{+}\right)$. Note that $M_{1}$ is a 3-dimensional manifold with boundary, the boundary of $M_{1}$ being $\partial M_{1}=\partial B^{-} \cup \partial B^{+}$, disjoint union of a pair of two spheres, which can be considered as $\mathbb{S}^{2} \cup \mathbb{S}^{2}$, where we will use $\mathbb{S}^{2}$ to denote various copies of the twodimensional unit sphere. The two closest points of $\partial B^{-}$ and $\partial B^{+}, P^{-}$and $P^{+}$, can be identified with the north pole of each sphere, $N P \in \mathbb{S}^{2}$.

The second component of $M$ is a 3-dimensional cylinder, $M_{2}=\mathbb{S}^{2} \times[0, L]$, that is, the product of sphere and an interval $[0, L] \subset \mathbb{R}$. As the boundaries of $M_{1}$ and $M_{2}$ are topologically the same, we can glue their boundaries together. The resulting domain $M$ no longer lies in $\mathbb{R}^{3}$, but rather has the topology of Euclidian space with a 3 -dimensional handle attached. $M$ is in fact a three dimensional manifold (without boundary) that is the connected sum of the components $M_{1}$ and $M_{2}$ (Fig. 1). On $M$, we use the Riemannian metric $g$ that is the Euclidian metric on $M_{1}$ and the product metric on $M_{2}$.

To consider Maxwell's equations, we start with Maxwell's equations on $\mathbb{R}^{3}$ at frequency $k \in \mathbb{R}$,

$\nabla \times E=i k B, \quad \nabla \times H=-i k D, \quad D=\varepsilon E, \quad B=\mu H$.

Here, $\varepsilon$ and $\mu$ are matrices corresponding to permittivity and permeability. Considering $E$ and $H$ as 1-forms and $D$ and $B$ as 2-forms, and using the exterior derivative $d$, Maxwell's equations can be written in coordinate invariant form as

$$
d E=i k B, \quad d H=-i k D, \quad D=\epsilon E, \quad B=\mu H,
$$

where $\varepsilon$ and $\mu$ are linear operators mapping 1-forms to 2 -forms [16]. We define Maxwell's equations on the manifold $M$ as equations (2) with $\varepsilon$ and $\mu$ acting via the Hodge operator of $(M, g)[16,17]$.

Construction of the wormhole device $N$ in $\mathbb{R}^{3}$. -We next explain how to build a "device" $N$ in $\mathbb{R}^{3}$ which affects the propagation of electromagnetic waves in the same way as the presence of the handle $M_{2}$ in the wormhole manifold $M$. We emphasize that we are not actually tearing and gluing space, but instead prescribing EM parameter fields (which can be physically realized using metamaterials), which make the EM waves in $\mathbb{R}^{3}$ behave as if they were propagating on the wormhole manifold $M$. In other words, as far as EM observations of the wormhole device are concerned, it appears as if the topology of space has been changed.

For simplicity, we construct a device that has rotational symmetry about a line in $\mathbb{R}^{3}$. We use cylindrical coordinates $(\theta, r, z)$ corresponding to a point $(r \cos \theta$, $r \sin \theta, z)$ in $\mathbb{R}^{3}$. The wormhole device is built around an 


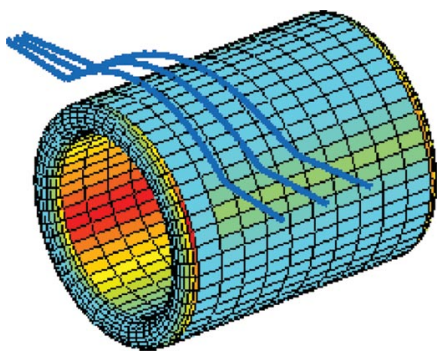

FIG. 3 (color). Obstacle $K$ and light rays going around the wormhole. The exterior $N$ of $K$ consists of two components; the bore $N_{2}$ of the wormhole device, and the outside $N_{1}$.

obstacle $K \subset \mathbb{R}^{3}$. To define $K$, let $S$ be the twodimensional finite cylinder $\{\theta \in[0,2 \pi], r=2,0 \leq z \leq$ $s\} \subset \mathbb{R}^{3}$. The closed region $K$ is a tubular neighborhood of $S$ and has the shape of a thickened cylinder with smoothed edges; see Figs. 3 and 4.

Using coordinate invariance of Maxwell's equations, we prove that there is a $1-1$ correspondence between the solutions of Maxwell's equations on $M$ and $N=\mathbb{R}^{3} \backslash K$ when the material parameters on $N$ are chosen appropriately. To describe those, let us consider a curve $\gamma$ that is the union of geodesic paths $\gamma_{1}$ and $\gamma_{2}$ that connect points $P^{+}$ and $P^{-}$in $M_{1}$ and $M_{2}$, respectively; see the red curves in Fig. 1. Then, there exists a diffeomorphism $F$ mapping $M \backslash$ $\gamma$ to $N$. To describe $F$, we use stereographic projection and a stretching in the $z$-coordinate to map $M_{2} \backslash \gamma_{2}=\left(\mathbb{S}^{2} \backslash\right.$ $\{N P\}) \times[0, L]$ onto $N_{2}=\{r<1,0 \leq z \leq s\}$ that is the product of a 2 -dimensional disc and an interval $[0, s]$ and forms the bore of the wormhole. Here, $L, s$ represent design parameters. The other component $M_{1} \backslash \gamma_{1}$ can be mapped to $N_{1}=N \backslash N_{2}$ so that the $\theta$-coordinate is preserved; we may further assume that this map is the identity far from the wormhole. The cross-section at $\theta=0$ of $F: M_{1} \backslash \gamma_{1} \rightarrow N_{1}$ is shown in Fig. 5. Note that $F$ blows up a neighborhood of $\gamma$ to a neighborhood of $\partial K$.

Maxwell's equations are invariant under smooth changes of coordinates. This means that any solution to Maxwell's equations on $M \backslash \gamma$ endowed with material parameters $\varepsilon, \mu$ becomes, after transformation by $F$, a solution to Maxwell's equations on $N$ with $F$-transformed material parameters $\tilde{\varepsilon}, \tilde{\mu}$, and vice versa. These correspond to an inhomogeneous, anisotropic medium that becomes singular as one approaches $\Sigma=\partial K$.

Next, consider the (light) rays in $M$ and in the exterior of the obstacle, $N=\mathbb{R}^{3} \backslash K$. The rays on $M$ are transformed by $F$ into the rays in $N$. As almost all the rays on $M$ do not intersect with $\gamma$, therefore, almost all the rays on $N$ do not approach $\Sigma$. This was the basis for [1,2] and was analyzed further in [3]; see also [12] for a similar analysis in the context of elasticity. Thus, heuristically, one is led to conclude that the electromagnetic waves on $(M ; \varepsilon, \mu)$ do not feel the presence of $\gamma$, while those on $(N ; \tilde{\varepsilon}, \tilde{\mu})$ do not feel the presence of $K$, and these waves are transformed into each other by the map $F$.

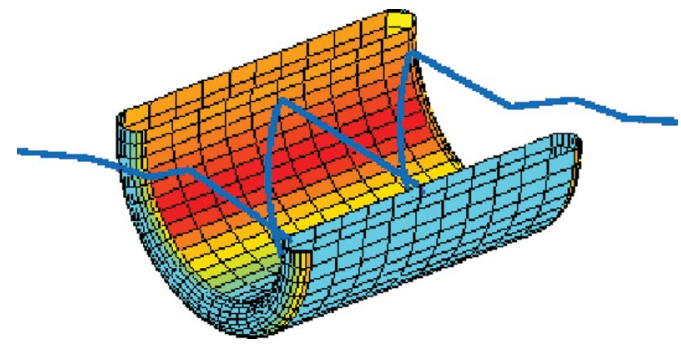

FIG. 4 (color). A typical light ray traversing the bore of the wormhole when $L \gg 1$. The upper part of $K$ is not shown.

However, when considering the fields on the entire spaces $M$ and $N$, neither the chain rule nor the ray-tracing analysis is adequate, due to the singularities of $\tilde{\varepsilon}$ and $\tilde{\mu}$ near $\Sigma$. The significance of this for cloaking has been analyzed in [5]. In the above construction, we removed a one-dimensional curve $\gamma$ from the manifold $M$. Removing $\gamma$ is analogous to making a one-dimensional crack that does not affect the EM fields. A careful analysis shows that the physically relevant class of waves, namely those that are locally finite energy and distributional solutions to Maxwell's equations on $(M ; \varepsilon, \mu)$ and $(N ; \tilde{\varepsilon}, \tilde{\mu})$, correspond perfectly under the transformation $F$. To guarantee that the fields in $N$ with singular material parameters $\tilde{\varepsilon}$ and $\tilde{\mu}$ are finite energy solutions and do not blow up near $\Sigma$, one must impose at $\Sigma$ the appropriate boundary condition, namely, the Soft-and-Hard (SH) condition, (see [18,19])

$$
\left.e_{\theta} \cdot E\right|_{\Sigma}=0,\left.\quad e_{\theta} \cdot H\right|_{\Sigma}=0
$$

where $e_{\theta}$ is the unit vector field in the angular direction.

Combining these steps, we see that external measurements of the electromagnetic fields on $(M ; \varepsilon, \mu)$ and on $\left(\mathbb{R}^{3} \backslash K ; \tilde{\varepsilon}, \tilde{\mu}\right)$ coincide. In other words, if we externally apply any EM wave and measure the radiating electromagnetic field it generates, then the field on the wormhole manifold $(M ; \varepsilon, \mu)$ coincides with the field on the wormhole device $\left(\mathbb{R}^{3} \backslash K ; \tilde{\varepsilon}, \tilde{\mu}\right)$.

Summarizing our constructions, the wormhole device consists of the ideal metamaterial coating of the obstacle $K$. This coating should have the permittivity $\tilde{\varepsilon}$ and permeability $\tilde{\mu}$. In addition, one should impose the SH boundary condition on $\Sigma$, which may be realized by making the obstacle $K$ from a perfectly conducting material with parallel corrugations on its surface $[18,19]$.

When the "length" of the wormhole is small, $L \ll 1$, the wormhole device results in an optical effect so that rays traversing the bore $N_{2}$ of the wormhole are acted on as by a fisheye lens, or a mirror ball with the image in the mirror

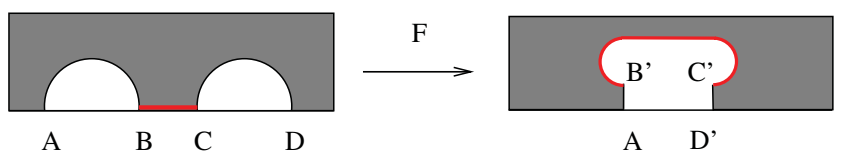

FIG. 5 (color). The map $F$ from $M_{1}$ to $N_{1}$ in $(r, z)$-coordinates. 
being from the other end of the wormhole; for $L \gtrsim 1$, further distortion and multiple images occur (Fig. 2).

For some potential applications, it is desirable to allow for a solid cylinder around the axis of the wormhole to consist of a vacuum or air, and it is possible to provide for that using a slightly different construction than was described above, starting with flattened spheres [14].

The proposed ideal material parameters $\varepsilon, \mu$ have, in cylindrical coordinates near $\partial K$, the same rotational symmetry as those used in [3] to theoretically cloak an infinite cylinder, see [14]. Thus, the metamaterial construction using concentric rings of split-ring resonators used for the experimental realization in [6] could be modified for an implementation of the wormhole device at a microwave frequency. We note that physical materials are always approximations of the ideal material parameters $\varepsilon, \mu$. The effect on cloaking of using $\varepsilon, \mu$ with materials having an anisotropy of limited magnitude has been studied in $[20,21]$, and similar analysis for the wormhole construction would be desirable.

Applications. -Finally, we consider applications of wormhole devices. The current rapid development of metamaterials designed for microwave and optical frequencies $[6,22,23]$ indicates the potential for physical applications of the wormhole construction, which are numerous:

Optical cables. - A wormhole device functions as an invisible optical tunnel or cable. In particular, a wormhole device, considered as an invisible tunnel, could be useful in making measurements of electromagnetic fields without disturbing those fields; these tunnels do not radiate energy to the exterior except from their ends.

Virtual magnetic monopoles. - Consider a very long invisible tunnel. Assume that one end of the tunnel is located in a region where a magnetic field enters the wormhole. Then, the other end of the tunnel behaves like a magnetic monopole, see [24].

Optical computers. - Wormholes could be used in optical computers. For instance, active components could be located inside wormhole devices having only visible "exits" for input and output.

$3 D$ video displays. -Divide a cube in $\mathbb{R}^{3}$ to $N \times N \times N$ voxels (three dimensional pixels) and put an end of an invisible tunnel into each voxel. Assume that the end of each tunnel is much smaller than the voxel so that from the exterior of the cube, all ends of the invisible tunnels are directly visible along any line that does not intersect the other ends of the wormholes. Then, by inserting light from the other ends of these $N^{3}$ invisible tunnels, one could direct light separately to each of the voxels. This creates a device acting as a "three dimensional video display."

Scopes for MRI devices. - We can modify construction of $M_{1}$ and $M_{2}$ by deforming the sphere $\mathbb{S}^{2}$ so that it is flat near the south pole $S P$ and the north pole $N P$ and making the tube $M_{2}$ longer. This then allows the permittivity $\tilde{\varepsilon}$ and permeability $\tilde{\mu}$ in $N$ to be constant near the $z$-axis. This means that inside the wormhole, there could be vacuum or air. Thus, for instance, in Magnetic Resonance Imaging
(MRI), we could use a wormhole to build a tunnel that would not disturb the homogeneous magnetic field needed for the imaging. Through such a tunnel, or "scope," magnetic metals and other materials or components can be transported to the imaged area without disturbing the fields. Such tunnels could be useful in medical operations using simultaneous MRI imaging.

Wormholes for beam collimation. - Consider a wormhole with a warped product metric on $M_{2}$, i.e., that at a point, $(y, u) \in \mathbb{S}^{2} \times[0,1]$ is the product of the standard metric of sphere $\mathbb{S}_{r(u)}^{2}$ of radius $r(u)$ and $d z$. Making $r(u)$ very small in the middle of the wormhole, only the rays that travel almost parallel to the axis of the wormhole can pass through it.

A. G. and G. U. are supported by US NSF, M. L. by the Academy of Finland.

*Authors are in alphabetical order.

[1] U. Leonhardt, Science 312, 1777 (2006).

[2] J. B. Pendry, D. Schurig, and D. R. Smith, Science 312, 1780 (2006).

[3] J. B. Pendry, D. Schurig, and D. R. Smith, Opt. Express 14, 9794 (2006).

[4] U. Leonhardt and T. Philbin, New J. Phys. 8, 247 (2006).

[5] A. Greenleaf et al., Commun. Math. Phys. 275, 749 (2007).

[6] D. Schurig et al., Science 314, 977 (2006).

[7] M. Lassas et al., Commun. Anal. Geom. 11, No. 2, 207 (2003).

[8] A. Greenleaf, M. Lassas, and G. Uhlmann, Physiol. Meas. 24, 413 (2003).

[9] A. Greenleaf, et al., Math. Res. Lett. 10, 685 (2003).

[10] A. Greenleaf, M. Lassas, and G. Uhlmann, Commun. Pure Appl. Math. 56, 328 (2003).

[11] G. Milton and N.-A. Nicorovici, Proc. R. Soc. A 462, 3027 (2006).

[12] G. Milton, M. Briane, and J. Willis, New J. Phys. 8, 248 (2006).

[13] S. Cummer and D. Schurig, New J. Phys. 9, 45 (2007).

[14] A. Greenleaf et al., arXiv:0704.0914.

[15] S. Hawking and G. Ellis, The Large Scale Structure of Space-Time (Cambridge University Press, Cambridge, England, 1973).

[16] A. Bossavit, Électromagnètisme, en Vue de la Modèlisation (Springer-Verlag, Berlin, 1993).

[17] Y. Kurylev et al., J. Math. Pures Appl. 86, 237 (2006).

[18] I. Hänninen, I. Lindell, and A. Sihvola, Prog. Electromagn. Res. PIER-64, 317 (2006).

[19] P.S. Kildal, IEEE Trans. Antennas Propag. 38, 1537 (1990).

[20] Z. Ruan et al., arXiv:0704.1183 [Phys. Rev. Lett. (to be published)].

[21] A. Greenleaf et al., Opt. Express 15, 12717 (2007).

[22] C. Soukoulis et al., Science 315, 47 (2007).

[23] W. Cai et al., Nat. Photon. 1, 224 (2007).

[24] T. Frankel, The Geometry of Physics (Cambridge University Press, Cambridge, England, 1997). 